\title{
INFINITE-DIMENSIONAL LIE GROUPS WITHOUT COMPLETENESS RESTRICTIONS
}

\author{
HELGE GLÖCKNER \\ Department of Mathematics, Louisiana State University, Baton Rouge, LA 70803-4918, U.S.A. \\ From May 2001: Math. Institut, Univ. Göttingen, Bunsenstr. 3-5, 37073 Göttingen, Germany \\ E-mail: gloeckne@uni-math.gwdg.de
}

\begin{abstract}
We describe a setting of infinite-dimensional smooth (resp., analytic) Lie groups modelled on arbitrary, not necessarily sequentially complete, locally convex spaces, generalizing the framework of Lie theory formulated in [R. Hamilton, The inverse function theorem of Nash and Moser, Bull. Amer. Math. Soc. 7 (1982), 65-222] for Fréchet modelling spaces and in [J. Milnor, Remarks on infinite-dimensional Lie groups, in: B. DeWitt and R. Stora (eds.), Relativity, Groups and Topology II, North-Holland, 1983] for sequentially complete modelling spaces. Our studies were dictated by the needs of infinite-dimensional Lie theory in the context of the existence problem of universal complexifications. We explain why satisfactory results in this area can only be obtained if the requirement of sequential completeness is abandoned.
\end{abstract}

Introduction. The Fundamental Theorem of Calculus, asserting that

$$
\gamma(b)-\gamma(a)=\int_{a}^{b} \gamma^{\prime}(t) d t
$$

for every $\mathcal{C}^{1}$-curve $\gamma:[a, b] \rightarrow E$ with values in a sequentially complete, locally convex (s.c.l.c.) space $E$, plays a central role in the theory of smooth mappings between s.c.l.c. spaces (as described in [15], for example). Since the Riemann integral $\int_{a}^{b} \gamma(t) d t$ exists for every continuous curve $\gamma:[a, b] \rightarrow E$ with values in an s.c.l.c. space (whereas this is not the case for general locally convex spaces $E$ ), the setting of s.c.l.c. spaces is certainly one natural possible framework for differential calculus, and most treatments of differential calculus impose sequential completeness or other completeness conditions on the locally convex spaces considered (sequential completeness in [15]; quasi-completeness in [18]; convergence of Mackey-Cauchy sequences in [14]). We remark that the second half of the ordinary Fundamental Theorem of Calculus generalizes to the setting of s.c.l.c. spaces as well: for every continuous curve $\gamma: I \rightarrow E$ defined on an interval around $t_{0}$ with

2000 Mathematics Subject Classification: Primary 58C20; Secondary 22E65, 46T20, 46T25.

Received 6 March 2001; revised 28 August 2001.

The paper is in final form and no version of it will be published elsewhere. 
values in $E$, we have $\gamma(t)=\frac{d}{d t} \int_{t_{0}}^{t} \gamma(s) d s$. Also the existence of complex line integrals of continuous functions with values in complex s.c.l.c. spaces is guaranteed, and these integrals play an important role in the theory of complex analytic functions on infinitedimensional spaces (see [4], [12]).

However, in the context of the existence problem of universal complexifications for infinite-dimensional Lie groups, as well as the existence problem of Lie group structures on quotient groups of Lie groups, it turned out that the framework of Lie groups modelled on sequentially complete spaces is too restrictive and prevents the formulation of results in their natural generality and simplicity. For example, a recent result in the theory of Banach-Lie groups says that the topological quotient group $G / N$ of a Banach-Lie group $G$ by a closed normal subgroup $N$ can be made a Banach-Lie group if and only if $N$ is a (not necessarily split) Lie subgroup of $G$ ([10], Corollary II.4). ${ }^{1}$ For a well-behaved class of infinite-dimensional analytic Lie groups whose group multiplication is given locally by the Baker-Campbell-Hausdorff series, called "BCH-Lie groups," an analogous characterization of the existence of BCH-Lie group structures on $G / N$ can be obtained ([8], Corollary 2.21). In contrast, in the setting of those BCH-Lie groups modelled on s.c.l.c. spaces, we are not allowed to consider $G / N$ as a Lie group when $N \leq G$ is a closed normal Lie subgroup for which $L(G) / L(N)$ fails to be sequentially complete, although the multiplication on $G / N$ is still given locally by the Campbell-Hausdorff series. This seems utterly unnatural. Similar unnatural (and superfluous) restrictions occur in the study of universal complexifications of those BCH-Lie groups which are modelled on s.c.l.c. spaces. All of these problems disappear immediately once the hypothesis of sequential completeness is abandoned. This experience finally convinced the author that Lie groups modelled on arbitrary locally convex spaces are necessities, not pathologies.

In this article, we present the basic theory of such groups (both smooth and analytic ones), and the underlying differential calculus. Here, the first essential observation is that only one half of the Fundamental Theorem of Calculus (the one stated in Equation(1) above) is actually needed to make differential calculus work. The second very simple, but essential observation is that no appeal to sequential completeness needs to be made to establish the existence of the relevant integrals: the integral in Equation(1) exists because the left hand side of Equation (1) is that integral (see also [13], Appendix). Having observed this, all basic results of differential calculus as compiled in Milnor [15] in the sequentially complete case carry over to the general case (Section 1). We remark that the concept of differentiability used here goes back to Michal and Bastiani ([2], cf. also [5]). For a comparative study of this and various other notions of differentiability, the reader is referred to Keller [13] (where sequential completeness is not assumed either). Equivalents of many of the results assembled in Section 1 can also be found in this more specialized text. Still, readers mainly interested in Lie groups may find Section 1 useful as it provides a brief, self-contained exposition of selected results particularly useful for infinite-dimensional Lie theory.

In Section 2 we discuss real and complex analytic mappings between open subsets of arbitrary locally convex spaces. We follow Bochnak and Siciak [3], [4] in the complex

\footnotetext{
${ }^{1}$ Note that $L(N)$ need not be complemented in $L(G)$ here.
} 
analytic case here but combine their ideas and Milnor's in the real analytic case. Again, it doesn't cause any problems to sacrifice sequential completeness.

Having established the foundations of smooth and analytic differential calculus, we can define smooth manifolds, analytic manifolds, and Lie groups modelled on arbitrary locally convex spaces, in the usual way. All of the basic results of Lie theory (as formulated by Milnor) carry over to the general case (Section 3).

We then turn to the class of BCH-Lie groups, which subsumes all Banach-Lie groups, loop groups, and "linear" direct limit Lie groups (Section 4). We discuss the above problems whose solutions essentially depend on the availability of differential calculus for arbitrary locally convex spaces, and which formed the stimulus for our investigations.

1. The differential calculus of smooth mappings. In this section, we describe the differential calculus of smooth mappings between open subsets of arbitrary locally convex spaces. Throughout the following, $\mathbb{K} \in\{\mathbb{R}, \mathbb{C}\}$.

We begin with the definition of curves.

Definition 1.1. A $\mathcal{C}^{0}$-curve is a continuous mapping $\gamma: I \rightarrow E$, where $E$ is a locally convex space and $I \subseteq \mathbb{R}$ an open interval. The $\mathcal{C}^{0}$-curve $\gamma$ is called a $\mathcal{C}^{1}$-curve if $\gamma^{\prime}(t):=$ $\lim _{s \rightarrow 0} s^{-1}(\gamma(t+s)-\gamma(t))$ exists for all $t \in I$ and $\gamma^{\prime}: I \rightarrow E$ is continuous.

REMARK 1.2. Note that $\phi \circ \gamma$ is a $\mathcal{C}^{1}$-curve, for every $\mathcal{C}^{1}$-curve $\gamma: I \rightarrow E$ and continuous linear map $\phi: E \rightarrow F$ from $E$ into a locally convex space $F$. We have $(\phi \circ \gamma)^{\prime}=\phi \circ \gamma^{\prime}$.

At the root of our studies is the observation that the essential portion of the Fundamental Theorem of Calculus for $\mathcal{C}^{1}$-curves with values in a locally convex space is valid irrespective of sequential completeness of the space.

This becomes most obvious if we use the weak definition of integrals:

Definition 1.3. Let $E$ be a locally convex space over $\mathbb{K}$, let $f: I \rightarrow E$ be a continuous function on an interval $I \subseteq \mathbb{R}$, and $a, b \in I$. If there exists an element $v_{0} \in E$ such that

$$
\left(\forall \lambda \in E^{\prime}\right) \quad \lambda\left(v_{0}\right)=\int_{a}^{b} \lambda(f(s)) d s,
$$

then we write $\int_{a}^{b} f(s) d s:=v_{0}$ and say that this integral exists.

REMARK 1.4. Note that $v_{0}$ as above is uniquely determined whenever it exists.

We can now prove without difficulty:

THEOREM 1.5 (Fundamental Theorem of Calculus). Let $E$ be a locally convex space, $I \subseteq \mathbb{R}$ be an open interval containing 0 , and $\gamma: I \rightarrow E$ be a $\mathcal{C}^{1}$-curve. Then the integral $\int_{0}^{t} \gamma^{\prime}(s) d s$ exists for all $t \in I$, and $\gamma(t)=\gamma(0)+\int_{0}^{t} \gamma^{\prime}(s) d s$.

Proof. Let $t \in I$. For every $\lambda \in E^{\prime}$, we have

$$
\lambda(\gamma(t)-\gamma(0))=\lambda(\gamma(t))-\lambda(\gamma(0))=\int_{0}^{t}(\lambda \circ \gamma)^{\prime}(s) d s=\int_{0}^{t} \lambda\left(\gamma^{\prime}(s)\right) d s
$$

in view of Remark 1.2, using the ordinary Fundamental Theorem of Calculus for functions $I \rightarrow \mathbb{K}$. Hence $\gamma(t)-\gamma(0)$ satisfies the defining property of the integral $\int_{0}^{t} \gamma^{\prime}(s) d s$, which therefore exists. The desired formula is apparent. 
REMARK 1.6. Clearly, the weak integral $\int_{a}^{b} f(t) d t$ in Definition 1.3 exists if and only if the corresponding Riemann integral exists (using that the latter always exists in the completion of $E$ ). For our purposes, the point of view of weak integrals is more convenient.

For later use, we recall a useful fact concerning weak integrals, which is an immediate consequence of the Bipolar Lemma:

LEMMA 1.7. Let $E$ be a locally convex space over $\mathbb{K}$ and $f:[a, b] \rightarrow E$ be a continuous function. Suppose that the weak integral $\int_{a}^{b} f(t) d t$ exists in E. Then

$$
q\left(\int_{a}^{b} f(t) d t\right) \leq(b-a) \sup \{q(f(t)): t \in[a, b]\},
$$

for every continuous seminorm $q$ on $E$.

Next, let us define and analyze smooth maps between general locally convex spaces.

Definition 1.8. Suppose that $E$ and $F$ are real locally convex spaces, $U$ an open subset of $E$, and $f: U \rightarrow F$ a continuous map. We say that $f$ is of class $\mathcal{C}^{1}$ if the (two-sided) directional derivative

$$
d f(x ; h):=\lim _{t \rightarrow 0} t^{-1}(f(x+t h)-f(x))
$$

exists for all $x \in U$ and $h \in E$ (where $t \in \mathbb{R} \backslash\{0\}$ with $x+t h \in U$ ), and if $d f: U \times E \rightarrow F$ is a continuous map. Recursively, we define higher derivatives via

$$
d^{n} f\left(x ; h_{1}, \ldots, h_{n}\right):=\lim _{t \rightarrow 0} \frac{1}{t}\left(d^{n-1} f\left(x+t h_{n} ; h_{1}, \ldots, h_{n-1}\right)-d^{n-1} f\left(x ; h_{1}, \ldots, h_{n-1}\right)\right)
$$

provided that all limits involved exist, and say that $f$ is of class $\mathcal{C}^{n}$ if $d^{k} f: U \times E^{k} \rightarrow F$ exists for all $k=1, \ldots, n$ and is continuous. The mapping $f$ is called smooth (or of class $\left.\mathcal{C}^{\infty}\right)$ if it is of class $\mathcal{C}^{n}$ for all $n \in \mathbb{N}$. Throughout the following, $r \in \mathbb{N} \cup\{\infty\}$.

Lemma 1.9. Suppose that $E$ and $F$ are real locally convex spaces, $U$ is an open subset of $E, f: U \rightarrow F$ a mapping of class $\mathcal{C}^{1}$, and $x_{0} \in U$. Then

$$
d f\left(x_{0} ; \bullet\right): E \rightarrow F, \quad h \mapsto d f\left(x_{0} ; h\right)
$$

is a continuous linear map.

Proof. Clearly $d f\left(x_{0} ; r v\right)=r d f\left(x_{0} ; v\right)$ for all $v \in E$ and $r \in \mathbb{R}$. Now suppose that $v, w \in E$; then there is $\delta>0$ such that $x_{0}+r v+s w \in U$ for all $r, s \in \mathbb{R}$ such that $|r|$, $|s| \leq \delta$. Using Theorem 1.5 twice, we calculate for $|t| \leq \delta$ :

$$
\begin{aligned}
f\left(x_{0}+\right. & t(v+w)) \\
= & f\left(x_{0}+t v\right)+\int_{0}^{1} d f\left(x_{0}+t v+s t w ; t w\right) d s \\
= & f\left(x_{0}\right)+\int_{0}^{1} d f\left(x_{0}+s t v ; t v\right) d s+\int_{0}^{1} d f\left(x_{0}+t v+s t w ; t w\right) d s \\
= & f\left(x_{0}\right)+t\left(d f\left(x_{0} ; v\right)+d f\left(x_{0} ; w\right)\right) \\
& \quad+t \int_{0}^{1}\left[d f\left(x_{0}+s t v ; v\right)-d f\left(x_{0} ; v\right)+d f\left(x_{0}+t v+s t w ; w\right)-d f\left(x_{0} ; w\right)\right] d s
\end{aligned}
$$


note that the integral in the last line exists, as its integrand is a sum of integrands for which the integrals exist. ${ }^{2}$ Now suppose that a continuous seminorm $q$ on $F$ and $\varepsilon>0$ are given. By continuity of $d f(\bullet ; v)$ and $d f(\bullet ; w)$, there exists a convex zero-neighbourhood $W$ in $E$ such that $x_{0}+W \subseteq U$ and $q\left(d f(x ; v)-d f\left(x_{0} ; v\right)\right) \leq \frac{\varepsilon}{2}, q\left(d f(x ; w)-d f\left(x_{0} ; w\right)\right) \leq \frac{\varepsilon}{2}$ for all $x \in x_{0}+W$. There is $0<\delta_{0} \leq \delta$ such that $r v+s w \in W$ for all $s, t \in \mathbb{R}$ with $|s|,|t| \leq \delta_{0}$. Then for any $t \in \mathbb{R}$ such that $0<|t|<\delta_{0}$, we deduce from the displayed formulas and Lemma 1.7 that $q\left(t^{-1}\left[f(x+t(v+w))-f\left(x_{0}\right)\right]-\left[d f\left(x_{0} ; v\right)+d f\left(x_{0} ; w\right)\right]\right) \leq \varepsilon$. We have proved that $\lim _{t \rightarrow 0} t^{-1}\left(f(x+t(v+w))-f\left(x_{0}\right)\right)=d f\left(x_{0} ; v\right)+d f\left(x_{0} ; w\right)$.

To ensure that a mapping on a product is of class $\mathcal{C}^{1}$, we only need to establish existence and continuity of the partial derivatives.

Lemma 1.10. Let $E, F$, and $G$ be real locally convex spaces, and let $U \subseteq E$ and $V \subseteq F$ be open subsets. Suppose that $f: U \times V \rightarrow G$ is a continuous map such that $d f(x ; v)$ exists for all $x \in U \times V$ and $v \in E \times\{0\} \cup\{0\} \times F$, and suppose that the mappings

$$
(U \times V) \times E \rightarrow G, \quad(x, v) \mapsto d f(x ;(v, 0))
$$

and

$$
(U \times V) \times F \rightarrow G, \quad(x, w) \mapsto d f(x ;(0, w))
$$

are continuous. Then $f$ is of class $\mathcal{C}^{1}$.

Proof. The proof of Lemma 1.9 shows that $d f(x ;(v, w))$ exists for all $(v, w) \in E \times F$ and $x \in U \times V$, and that it is given by $d f(x ;(v, w))=d f(x ;(v, 0)+(0, w))=d f(x ;(v, 0))+$ $d f(x ;(0, w))$. In view of the continuity hypothesis made in the lemma, we deduce from the preceding formula that $d f$ is continuous. Thus $f$ is of class $\mathcal{C}^{1}$.

Proposition 1.11. Suppose that $f: U \rightarrow F$ is a mapping of class $\mathcal{C}^{1}$, where $F$ is a real locally convex space and $U$ an open subset of a real locally convex space $E$. If $d f=0$ on $U \times E$, then $f$ is constant on each connected component of $U$.

Proof. Making use of the Hahn-Banach Theorem, we can reduce to the case where $F=\mathbb{R}$. Every point $x_{0} \in U$ has an open star-shaped (or even convex) neighbourhood $V$ in $U$. We readily deduce from the well-known one-dimensional case that $f$ is constant on $V$. Hence $f$ is locally constant and therefore constant on each connected component of $U$.

Here is our first version of the Chain Rule.

Proposition 1.12. Suppose that E, $F$ and $G$ are real locally convex spaces, and suppose that $f: U \rightarrow V$ and $g: V \rightarrow G$ are mappings of class $\mathcal{C}^{1}$, where $U \subseteq E$ and $V \subseteq F$ are open subsets. Then $g \circ f: U \rightarrow G$ is a mapping of class $\mathcal{C}^{1}$, and

$$
(\forall x \in U ; \forall v \in E) \quad d(g \circ f)(x ; v)=d g(f(x) ; d f(x ; v)) .
$$

Proof. Note first that the mapping $g \circ f$ is continuous as a composition of continuous maps. If we can show that (2) holds, then clearly $d(g \circ f)$ will be continuous. So, let $x \in U$ and $v \in E$. There is $\delta>0$ such that $x+[-\delta, \delta] v \subseteq U$. As $d f(x ; v)$ exists, we have

$$
(g \circ f)(x+t v)=g(f(x)+t[d f(x ; v)+r(t)])
$$

\footnotetext{
${ }^{2}$ This argument will be used ever and ever again, without further mention.
} 
for all $t \in[-\delta, \delta]$, where $r:[-\delta, \delta] \rightarrow F$ is a continuous function with $r(0)=0$. We abbreviate $w(t):=d f(x ; v)+r(t)$. Shrinking $\delta$ if necessary, we may assume that $f(x)+$ st $w(t) \in V$ for all $t \in[-\delta, \delta]$ and all $s$ in an open interval $I$ containing [0,1]. It is apparent from the definition of mappings of class $\mathcal{C}^{1}$ that $\gamma: I \rightarrow G, \gamma(s):=g(f(x)+t s w(t))$ is a $\mathcal{C}^{1}$-curve; we have $\gamma^{\prime}(s)=d g(f(x)+t s w(t) ; t w(t))=t d g(f(x)+t s w(t) ; w(t))$. The Fundamental Theorem of Calculus (Theorem 1.5) yields

$$
\begin{aligned}
(g \circ f) & (x+t v)=\gamma(1)=\gamma(0)+\int_{0}^{1} \gamma^{\prime}(s) d s \\
= & g(f(x))+t d g(f(x) ; d f(x ; v)) \\
& \quad+t \int_{0}^{1}[d g(f(x)+t s w(t), d f(x ; v)+r(t))-d g(f(x) ; d f(x ; v))] d s \\
= & g(f(x))+t d g(f(x) ; d f(x ; v)) \\
& \quad+t \int_{0}^{1}[d g(f(x)+t s w(t), d f(x ; v))+d g(f(x)+t s w(t) ; r(t))-d g(f(x) ; d f(x ; v))] d s .
\end{aligned}
$$

Now suppose that $q$ is any continuous seminorm on $G$, and $\varepsilon>0$. In view of the compactness of $[0,1]$ we deduce from the continuity of the mappings

$$
\Phi:[-\delta, \delta] \times[0,1] \rightarrow G, \quad \Phi(t, s)=d g(f(x)+t s w(t) ; r(t))
$$

and

$$
\Psi:[-\delta, \delta] \times[0,1] \rightarrow G, \quad \Psi(t, s)=d g(f(x)+t s w(t), d f(x ; v))-d g(f(x) ; d f(x ; v))
$$

satisfying $\Phi(0, \bullet)=0$ and $\Psi(0, \bullet)=0$, that there exists $0<\delta_{0}<\delta$ such that $q(\Phi(t, s)) \leq \frac{\varepsilon}{2}$ and $q(\Psi(t, s)) \leq \frac{\varepsilon}{2}$ for all $t \in\left[-\delta_{0}, \delta_{0}\right]$ and $s \in[0,1]$. We deduce from Lemma 1.7 and the above longish formulas for $(g \circ f)(x+t v)$ that

$$
q\left(\frac{1}{t}(g(f(x+t v))-g(f(x)))-d g(f(x) ; d f(x ; v))\right) \leq \varepsilon
$$

for all $t \in \mathbb{R}$ with $0<|t|<\delta_{0}$. We have proved that $\lim _{t \rightarrow 0} t^{-1}(g(f(x+t v))-g(f(x)))=$ $d g(f(x) ; d f(x ; v))$.

Proposition 1.13. Suppose that $f: U \rightarrow F$ is a mapping of class $\mathcal{C}^{r}$, where $F$ is a real locally convex space and $U$ an open subset of a real locally convex space $E$. Then

$$
d^{k} f(x ; \bullet): E^{k} \rightarrow F, \quad\left(v_{1}, \ldots, v_{k}\right) \mapsto d f\left(x ; v_{1}, \ldots, v_{k}\right)
$$

is a symmetric, continuous, $k$-linear mapping, for every $x \in U$ and $k \in \mathbb{N}$ such that $k \leq r$.

Proof. We may assume that $k=r$. It is immediate from the definition of mappings of class $\mathcal{C}^{k}$ that $d^{k} f(x ; \bullet)$ is continuous. In view of the Hahn-Banach Theorem, the remaining assertions will follow if we can prove them in the case $F=\mathbb{R}$. It is also clear that $d^{k} f(x ; \bullet)$ will be a symmetric $k$-linear map if we can show that $d^{k}\left(\left.f\right|_{E_{0} \cap U}\right)(x ; \bullet)$ is a symmetric $k$-linear map for all finite-dimensional subspaces $E_{0}$ of $E$ containing $x$. Thus $\operatorname{dim}(E)<\infty$ without loss of generality. For $\mathcal{C}^{k}$-mappings between open subsets of finite-dimensional spaces however, the assertion is well-known. 
Our next goal is to show that compositions of $\mathcal{C}^{r}$-maps are $\mathcal{C}^{r}$-maps. We shall obtain this important fact as an immediate consequence of the following technical lemma. Only for internal use in this article, let us agree on the following terminology. Given a function $f: U \rightarrow F$, where $U$ is an open subset of a locally convex space $E$ and $F$ is a locally convex space, let us say that $f$ is of class $\tilde{\mathcal{C}}^{1}$ if it is of class $\mathcal{C}^{1}$, and set $D f:=d f$. Inductively, let us say that $f$ is of class $\tilde{\mathcal{C}}^{k+1}$ if $f$ is of class $\tilde{\mathcal{C}}^{k}$ and $D^{k} f$ is of class $\mathcal{C}^{1}$. We set $D^{k+1} f:=D\left(D^{k} f\right)$ in this case. Thus $D f: U \times E \rightarrow F, D^{2} f:(U \times E) \times(E \times E) \rightarrow F$, and in general $D^{k} f: U \times E^{2^{k}-1} \rightarrow F$, noting that $1+2+2^{2}+\cdots+2^{k-1}=2^{k}-1$.

Lemma 1.14. Let $E$ and $F$ be real locally convex spaces, $f: U \rightarrow F$ be a continuous function defined on an open subset $U$ of $E$, and $k \in \mathbb{N}$. Then $f$ is of class $\mathcal{C}^{k}$ if and only if $f$ is of class $\tilde{\mathcal{C}}^{k}$.

Proof. Given $n \in \mathbb{N}$, we abbreviate $\mid n]:=\{1,2, \ldots, n\}$.

Claim 1. If $f$ is of class $\mathcal{C}^{k}$, then $f$ is of class $\tilde{\mathcal{C}}^{k}$. For $i=1, \ldots, k$, there are functions $\left.n_{\bullet}^{i}: \mid 2^{k}-1\right]^{\mid i]} \rightarrow \mathbb{N}_{0}$ such that, for all $x \in U$ and $v=\left(v_{1}, \ldots, v_{2^{k}-1}\right) \in E^{2^{k}-1}$,

$$
\left(D^{k} f\right)(x ; v)=\sum_{i=1}^{k} \sum_{\alpha \in\left[2^{k}-1\right]^{\mid i]}} n_{\alpha}^{i} d^{i} f\left(x ; v_{\alpha(1)}, \ldots, v_{\alpha(i)}\right) .
$$

We prove the claim by induction. By definition, $f$ is of class $\mathcal{C}^{1}$ if and only if $f$ is of class $\tilde{\mathcal{C}}^{1}$, and $D f=d f$. Thus the claim holds if $k=1$.

Induction Step. Suppose that $k \geq 1$ and suppose that $f$ is of class $\mathcal{C}^{k+1}$. Then $f$ is of class $\mathcal{C}^{k}$ as well; by induction hypothesis, $f$ is of class $\tilde{\mathcal{C}}^{k}$, with $D^{k} f$ of the form described in Equation (3). Now, since $f$ is of class $\mathcal{C}^{k+1}$, it is apparent from Equation (3) that $d\left(D^{k} f\right)((x, v) ;(w, 0))$ exists for all $(x, v) \in U \times E^{2^{k}-1}$ and $w \in E$; it is given by

$$
d\left(D^{k} f\right)((x, v),(w, 0))=\sum_{i=1}^{k} \sum_{\left.\alpha \in \mid 2^{k}-1\right]^{\mid i]}} n_{\alpha}^{i} d^{i+1} f\left(x ; v_{\alpha(1)}, \ldots, v_{\alpha(i)}, w\right),
$$

which is a continuous function of $(x, v, w) \in U \times E^{2^{k}}$. On the other hand, it follows from the $i$-linearity of the mappings $d^{i} f(x ; \bullet)$ and Equation $(3)$ that $d\left(D^{k} f\right)((x, v),(0, z))$ exists, for all $(x, v) \in U \times E^{2^{k}-1}$ and $z=\left(z_{1}, \ldots, z_{2^{k}-1}\right) \in E^{2^{k}-1}$; it is a sum of the directional derivatives of mappings of the form $E^{2^{k}-1} \rightarrow F, u \mapsto d^{i} f\left(x ; u_{\alpha(1)}, \ldots, u_{\alpha(i)}\right)$, in the direction $z$ at $v$. A function of the latter form being a composition of an $i$-linear map and a linear map (namely, $u \mapsto\left(u_{\alpha(1)}, \ldots, u_{\alpha(i)}\right)$ ), the required directional derivative has the form $d^{i} f\left(x ; z_{\alpha(1)}, v_{\alpha(2)}, \ldots, v_{\alpha(i)}\right)+\cdots+d^{i} f\left(x ; v_{\alpha(1)}, \ldots, v_{\alpha(i-1)}, z_{\alpha(i)}\right)$; note that this is a continuous function of $(x, v, z)$. In view of Lemma 1.10, we deduce from (4) and the preceding consideration that $d\left(D^{k} f\right)=: D^{k+1} f$ exists, is continuous, and is given by

$$
\begin{aligned}
& D^{k+1} f((x, v) ;(w, z))=\sum_{i=1}^{k} \sum_{\left.\alpha \in \mid 2^{k}-1\right]^{\mid i]}} n_{\alpha}^{i} d^{i+1} f\left(x ; v_{\alpha(1)}, \ldots, v_{\alpha(i)}, w\right) \\
& \quad+\sum_{i=1}^{k} \sum_{\left.\alpha \in \mid 2^{k}-1\right]^{\mid i]}} n_{\alpha}^{i} \sum_{j=1}^{i} d^{i} f\left(x ; v_{\alpha(1)}, \ldots, v_{\alpha(j-1)}, z_{\alpha(j)}, v_{\alpha(j+1)}, \ldots, v_{\alpha(i)}\right),
\end{aligned}
$$

which can easily be brought to the form (3) (with $k$ replaced by $k+1$ ). 
Claim 2. If $f$ is of class $\tilde{\mathcal{C}}^{k}$, then $f$ is of class $\mathcal{C}^{k}$. Furthermore, there is an injective function $\left.\gamma: \mid k] \rightarrow \mid 2^{k}-1\right]$ such that

$$
\left(\forall x \in U, \forall v=\left(v_{1}, \ldots, v_{k}\right) \in E^{k}\right) \quad d^{k} f(x ; v)=\left(D^{k} f\right)(x ; \phi(v)),
$$

where $\phi: E^{k} \rightarrow E^{2^{k}-1}$ is the mapping determined by

$$
\left(\forall j=1, \ldots, 2^{k}-1\right) \quad \operatorname{pr}_{j}\left(\phi\left(v_{1}, \ldots, v_{k}\right)\right)= \begin{cases}v_{i} & \text { if } \gamma(i)=j \\ 0 & \text { else. }\end{cases}
$$

Again, the proof is by induction. If $k=1$, we have $d f(x ; v)=D f(x ; v)=D f(x ; \phi(v))$ with $\gamma:=\mathrm{id}: \mid 1] \rightarrow \mid 1]$ and $\phi=\mathrm{id}_{E}$.

Induction Step. Suppose that $f$ is of class $\tilde{C}^{k+1}$, where $k \geq 1$. Then $f$ is of class $\tilde{\mathcal{C}}^{k}$ and therefore of class $\mathcal{C}^{k}$ by induction hypothesis; furthermore, we have $d^{k} f(x ; v)=$ $\left(D^{k} f\right)(x, \phi(v))$ for all $(x, v) \in U \times E^{k}$ for a certain $\phi$ (and $\gamma$ ) as above. Thus, given $(x, v) \in U \times E^{k}$ and $v_{k+1} \in E$, clearly

$$
\begin{aligned}
t^{-1}\left(d^{k} f\left(x+t v_{k+1} ; v\right)-d^{k} f(x ; v)\right) & =t^{-1}\left(D^{k} f\left(x+t v_{k+1} ; \phi(v)\right)-D^{k} f(x ; \phi(v))\right) \\
& \rightarrow D^{k+1} f\left((x, \phi(v)),\left(v_{k+1}, 0\right)\right)
\end{aligned}
$$

as $t \rightarrow 0$. We deduce that $d^{k+1} f\left(x ;\left(v, v_{k+1}\right)\right)$ exists: it is given by

$$
d^{k+1} f\left(x ;\left(v, v_{k+1}\right)\right)=D^{k+1} f\left((x, \phi(v)) ;\left(v_{k+1}, 0\right)\right)=D^{k+1} f(x, \check{\phi}),
$$

where $\check{\phi}: E^{k+1} \rightarrow E^{2^{k+1}-1}$ arises from some injection $\left.\left.\check{\gamma}: \mid k+1\right] \rightarrow \mid 2^{k+1}-1\right]$ in the desired way. It only remains to note that Equation (6) entails that $d^{k+1} f$ is continuous.

We immediately deduce:

Proposition 1.15. Let $E, F$, and $G$ be real locally convex spaces, $U$ and $V$ be open subsets of $E$ and $F$, respectively, and suppose that $f: U \rightarrow V$ and $g: V \rightarrow G$ are mappings of class $\mathcal{C}^{r}$. Then the composition $g \circ f: U \rightarrow G$ is of class $\mathcal{C}^{r}$ as well.

Proof. We may assume that $r \in \mathbb{N}$ without loss of generality. The proof is by induction. The case $r=1$ having been settled in Proposition 1.12 above, let us assume now that $r \geq 2$ and that compositions of $\mathcal{C}^{r-1}$-maps are mappings of class $\mathcal{C}^{r-1}$. Let $f, g$ be mappings of class $\mathcal{C}^{r}$ as described in the lemma. Then $g \circ f$ is of class $\mathcal{C}^{1}$ by Proposition 1.12, and $d(g \circ f)(x ; v)=d g(f(x) ; d f(x ; v))$ for $x \in U$ and $v \in E$. Note that $d g: V \times F \rightarrow G$ and $d f: U \times E \rightarrow F$ are mappings of class $\mathcal{C}^{r-1}$ here, by Lemma 1.14. Both $f$ and $d f$ being $\mathcal{C}^{r-1}$-maps, it is easy to see that so is $h: U \times E \rightarrow V \times F, h(x, v):=(f(x) ; d f(x ; v))$. Using the induction hypothesis, we see that $d(g \circ f)=(d g) \circ h$ is of class $\mathcal{C}^{r-1}$ and therefore of class $\tilde{\mathcal{C}}^{r-1}$, whence $g \circ f$ is of class $\tilde{\mathcal{C}}^{r}$. Making use of Lemma 1.14 again, we find that $g \circ f$ is of class $\mathcal{C}^{r}$ indeed.

It is convenient to introduce more terminology:

Definition 1.16. Suppose that $E$ and $F$ are real locally convex spaces, $U \subseteq E$ an open subset, and $f: U \rightarrow F$ a mapping of class $\mathcal{C}^{r}$; let $x \in U$. We define $\delta_{x}^{0} f(v):=f(x)$ and $\delta_{x}^{k} f(v):=d^{k} f(x ; v, \ldots, v)$ for all $v \in E$ and natural numbers $k \leq r$. The mapping $\delta_{x}^{k} f: E \rightarrow F$ is called the $k$ th Gateaux differential of $f$ at $x$.

We can now formulate Taylor's Theorem as follows: 
Proposition 1.17. Suppose that $f: U \rightarrow F$ is a mapping of class $\mathcal{C}^{r}$, where $U$ is an open subset of a real locally convex space $E$ and $F$ is a locally convex space. Then, for every $k \in \mathbb{N}_{0}$ such that $k<r$, we have

$$
f(x+h)=f(x)+\delta_{x}^{1} f(h)+\delta_{x}^{2} f(h) / 2+\cdots+\delta_{x}^{k} f(h) / k !+R
$$

for all $(x, h) \in U \times E$ such that $x+[0,1] h \subseteq U$, where the remainder term $R$ is given by

$$
R=\frac{1}{k !} \int_{0}^{1}(1-t)^{k} \delta_{x+t h}^{k+1} f(h) d t .
$$

Proof. The assertion can be reduced to the case where $E$ has finite dimension. Applying continuous linear functionals, we readily deduce from the classical multi-variable Taylor Theorem that the remainder term $f(x+h)-f(x)-\cdots-\delta_{x}^{k} f(h) / k$ ! satisfies the defining property of the weak integral (7).

The following simple fact will be useful later:

LEMMA 1.18. Let $E$ and $F$ be real locally convex spaces, $U \subseteq E$ be an open subset, and $f: U \rightarrow F$ be a mapping of class $\mathcal{C}^{r}$. Then $\operatorname{span}_{\mathbb{R}} \delta_{x}^{k} f(E)=\operatorname{span}_{\mathbb{R}} d^{k} f\left(\{x\} \times E^{k}\right)$ for all $x \in U$ and $k \in \mathbb{N}, k \leq r$.

Proof. This is an immediate consequence of [3], Theorem A.

2. Analytic mappings. Real and complex analytic mappings between open subsets of arbitrary locally convex spaces have already been defined by Bochnak and Siciak [4], who however formulated all of their results only in the case of sequentially complete target spaces. We shall use the concept of complex analytic mappings developed in [4], but a different notion of real analytic mappings, based on Milnor's ideas.

Definition 2.1. Suppose that $E$ and $F$ are complex locally convex spaces, and $U$ is an open subset of $E$. We say that a function $f: U \rightarrow F$ is complex analytic if it is continuous and for every $x \in U$, there exists a 0-neighbourhood $V$ in $E$ such that $x+V \subseteq U$ and

$$
f(x+h)=\sum_{n=0}^{\infty} \beta_{n}(h) \quad \text { for all } h \in V
$$

as a pointwise limit, where $\beta_{n}: E \rightarrow F$ is a continuous homogeneous polynomial over $\mathbb{C}$ of degree $n$, for each $n \in \mathbb{N}_{0}$ ([4], Definition 5.6).

Remark 2.2. Provided $F$ is sequentially complete in the preceding situation, it is well-known that the mapping $f$ is complex analytic if and only if it is complex differentiable on each affine line and continuous ([4], Theorems 6.2 and 3.1).

Definition 2.3. Let $E$ and $F$ be real locally convex spaces, $U$ be an open subset of $E$, and $f: U \rightarrow F$ be a mapping. Copying Milnor's definition in the sequentially complete case [15], we say that $f$ is real analytic if it extends to a complex analytic mapping $V \rightarrow F_{\mathbb{C}}$ on some open neighbourhood $V$ of $U$ in $E_{\mathbb{C}}$.

Real analyticity of a mapping $f: U \rightarrow F$ is a local property in the sense that real analyticity of $\left.f\right|_{U_{j}}$ for an open cover $\left(U_{j}\right)_{j \in J}$ of $U$ entails real analyticity of $f$. In fact, if $f$ is locally real analytic, then for any $x \in U$ we find open, convex neighbourhoods $V_{x}$ 
of $x$ in $U$ and $W_{x}$ of 0 in $E$ such that $\left.f\right|_{V_{x}}=\left.g_{x}\right|_{V_{x}}$ for some complex analytic mapping $g_{x}: N_{x}:=V_{x}+i W_{x} \rightarrow F_{\mathbb{C}}$. Given $x, y \in U$, the mappings $g_{x}$ and $g_{y}$ coincide on $V_{x} \cap V_{y}$ and hence on all of $N_{x} \cap N_{y}$ by the Identity Theorem ([4], Proposition 6.6 II). Thus $g:=\bigcup_{x \in U} g_{x}: \bigcup_{x \in U} N_{x} \rightarrow F_{\mathbb{C}}$ is a well-defined complex analytic mapping extending $f$.

Proposition 2.4. Every real or complex analytic mapping $f: U \rightarrow F$ as above is smooth.

Proof. For the proof, we may assume that $F$ is a complex locally convex space and that $f$ is complex analytic. Making use of Lemma 1.14, we deduce inductively from [4], Proposition 6.4 that $f$ is smooth as a mapping into the completion $\bar{F}$ of $F$; thus $d^{k} f: U \times E^{k} \rightarrow \bar{F}$ is defined for each $k \in \mathbb{N}$. Let $x \in U$ and suppose that $f$ has the local expansion (8) around $x$. Then $\beta_{k}=\frac{1}{k !} \delta_{x}^{k} f$ for each $k \in \mathbb{N}_{0}$ (see [4]). Lemma 1.18 yields $F \supset \operatorname{span}\left(\beta_{k}(E)\right)=\operatorname{span}\left(\delta_{x}^{k} f(E)\right)=\operatorname{span}\left(d^{k} f\left(x ; E^{k}\right)\right)$ for each $k \in \mathbb{N}$. Thus $d^{k} f$ maps into $F$ for each $k \in \mathbb{N}$, which readily entails that $f$ is smooth as a mapping into $F$.

Lemma 2.5. Suppose that $E$ and $F$ are complex locally convex spaces, and $f: U \rightarrow F$ is a mapping defined on an open subset of $E$. Then $f$ is complex analytic if and only if $f$ is smooth and the mapping $d f(x ; \bullet)=\delta_{x}^{1}: E \rightarrow F$ is complex linear for each $x \in U$.

Proof. If $f$ is complex analytic, with local expansion $f(x+h)=\sum_{k=0}^{\infty} \beta_{k}(h)$ around $x$, then $\delta_{x}^{1}=\beta_{1}$ is complex linear; furthermore, $f$ is smooth by Proposition 2.4. Conversely, suppose that $f$ is smooth and $\delta_{x}^{1}$ is complex linear for all $x \in U$. By [4], Theorem 3.1, Implication $\left(\mathrm{A}_{1}\right) \Rightarrow\left(\mathrm{A}_{5}\right)$ and loc. cit. Theorem 6.2 , Implication (iii) $\Rightarrow(\mathrm{i})$, the function $f$ is complex analytic as a mapping into the completion $\bar{F}$ of $F$. Given $x \in U$, we then have $f(x+h)=\sum_{k=0}^{\infty} \frac{1}{k !} \delta_{x}^{k}(h)$ for $h$ in some suitable zero-neighbourhood in $E$ (loc. cit., Proposition 5.5). Now $f$ being smooth as a mapping into $F$, we have $\delta_{x}^{k}(E) \subseteq F$ for each $k \in \mathbb{N}_{0}$. Therefore $f$ is complex analytic as a mapping into $F$.

Lemma 2.6. Suppose that $f: U \rightarrow F$ is a complex analytic mapping, where $F$ is a complex locally convex space and $U$ an open subset of a complex locally convex space $E$. Then $d f: U \times E \rightarrow F$ is complex analytic as well.

Proof. In fact, $d f$ is smooth since $f$ is so (Lemma 2.5, Lemma 1.14). Furthermore, $\delta_{x}^{1}(d f)$ is complex linear for all $x \in U \times E$, since $d f$ is complex analytic as a mapping into the completion of $F$, by [4], Proposition 6.4. Now apply Lemma 2.5.

Proposition 2.7. Let $U$ and $V$ be open subsets of the complex locally convex spaces $E$ and $F$, respectively, and let $f: U \rightarrow V$ and $g: V \rightarrow G$ be complex analytic mappings, where $G$ is a complex locally convex space. Then the composition $g \circ f$ is complex analytic.

Proof. The mapping $g \circ f$ is smooth by Proposition 1.15 and Proposition 2.4. By Proposition 1.12, we have $\delta_{x}^{1}(g \circ f)=\left(\delta_{f(x)}^{1} g\right) \circ\left(\delta_{x}^{1} f\right)$, which is a complex linear mapping as $\delta_{f(x)}^{1} g$ and $\delta_{x}^{1} f$ are complex linear by Lemma 2.5. Using the converse direction of Lemma 2.5, we deduce that $g \circ f$ is complex analytic.

Proposition 2.8. Compositions of real analytic mappings are real analytic.

Proof. This readily follows from Proposition 2.7 and the definition of real analyticity. 
2.9. We remark that if $E$ and $F$ are Fréchet spaces, then a mapping $f$ as in Definition 2.3 is real analytic in our sense (i.e., in Milnor's sense) if and only if it is real analytic in the sense of [4], Definition 5.6, i.e., if and only if it is continuous and admits local expansions into continuous homogeneous polynomials over $\mathbb{R}$, as in Equation(8) (cf. [4], Theorem 7.1). We favour Milnor's stronger definition of real analytic mappings here since their compositions are real analytic, whereas the corresponding assertion does not seem to be clear for real analytic mappings $f, g$ in the sense of Bochnak and Siciak, unless the spaces $E$ and $F$ containing the domains of $f$ and $g$ are Baire spaces (cf. [4], Theorem 7.3).

The following two propositions are very useful. First, we show that when an analytic map factors over a linear quotient map, then the induced map will be analytic as well.

Proposition 2.10. Let $E$ and $F$ be locally convex spaces over $\mathbb{K}, N$ be a closed vector subspace of $E$, and $q: E \rightarrow E / N=: E_{1}$ be the quotient map. Suppose that $f: U \rightarrow F$ is a $\mathbb{K}$-analytic (resp., smooth) function, defined on an open subset $U$ of $E$, such that $f=\left.f_{1} \circ q\right|_{U} ^{U_{1}}$ for some function $f_{1}: U_{1} \rightarrow F$ on $U_{1}:=q(U)$. Then $f_{1}$ is $\mathbb{K}$-analytic (resp., smooth).

Proof. We note first that

$$
\begin{aligned}
d f_{1}(q(x) ; q(v)) & =\lim _{t \rightarrow 0} t^{-1}\left[f_{1}(q(x+t v))-f_{1}(q(x))\right] \\
& =\lim _{t \rightarrow 0} t^{-1}[f(x+t v)-f(x)]=d f(x ; v)=\operatorname{Df}(x ; v)
\end{aligned}
$$

for all $(x, v) \in U \times E$, whence $D f_{1}=d f_{1}$ is defined on $U_{1} \times E_{1}$ and is continuous since

$$
D f_{1} \circ\left(\left.q\right|_{U} ^{U_{1}} \times q\right)=D f
$$

is continuous, where $\left.q\right|_{U} ^{U_{1}} \times q$ is an open surjection and therefore a quotient map. Note that the smooth mapping $D f$ factors to the mapping $D f_{1}$ on $(q \times q)(U \times E)$ by Equation (9). The apparent induction shows that $f_{1}$ is of class $\tilde{C}^{k}$ for each $k$, with $D^{k} f=D^{k} f_{1}$ 。 $\left(\left.q\right|_{U} ^{U_{1}} \times q^{2^{k}-1}\right)$. We deduce from Lemma 1.14 that $f_{1}$ is smooth. If $\mathbb{K}=\mathbb{C}$ and $f$ is complex analytic, then the Gateaux differential $\delta_{z}^{1} f_{1}$ is complex linear for all $z \in U_{1}$, as $\delta_{q(x)}^{1} f_{1} \circ q=\delta_{x}^{1} f$ for each $x \in U$ by Equation(9); Lemma 2.5 shows that $f_{1}$ is complex analytic.

Now suppose that $\mathbb{K}=\mathbb{R}$ and $f$ is real analytic. Then there exists a complex analytic mapping $g: V \rightarrow F_{\mathbb{C}}$ extending $f$, where $V$ is an open neighbourhood of $U$ in $E_{\mathbb{C}}$, and $V \cap E=U$. Given $x \in U$, there is a balanced 0-neighbourhood $W$ in $E_{\mathbb{C}}$ such that $x+W+W+W+W \subseteq V$. Given $w_{1}, w_{2} \in W$ and $n_{1}, n_{2} \in W \cap N$, we consider the mappings $h_{1}, h_{2}: \bar{D} \times \bar{D} \rightarrow F_{\mathbb{C}}$, defined via $h_{1}\left(z_{1}, z_{2}\right):=g\left(x+w_{1}+z_{1} w_{2}+n_{1}+z_{2} n_{2}\right)$ and $h_{2}\left(z_{1}, z_{2}\right):=g\left(x+w_{1}+z_{1} w_{2}\right)$; here $\bar{D} \subseteq \mathbb{C}$ is the closed unit disk. Then $h_{1}$ and $h_{2}$ are continuous, holomorphic on $D \times D$, and coincide on $[-1,1]^{2}$. Hence $h_{1}=h_{2}$ in view of [4], Proposition 6.6 II (Identity Theorem). Thus $g\left(x+w_{1}+i w_{2}+n_{1}+i n_{2}\right)=g\left(x+w_{1}+i w_{2}\right)$ in particular. There is an open 0-neighbourhood $A$ in $E$ such that $A-A \subseteq W$. Then $g(x+b)=g\left(x+b^{\prime}\right)$ whenever $b, b^{\prime} \in B:=A+i A$ such that $b-b^{\prime} \in N_{\mathbb{C}}$. Hence $\left.g\right|_{x+B}$ factors to a mapping $g_{1}: q_{\mathbb{C}}(x+B) \rightarrow F_{\mathbb{C}}$. As $g_{1}$ is complex analytic by the above, we deduce that $\left.f_{1}\right|_{q(x+A)}=\left.g_{1}\right|_{q(x+A)}$ is real analytic. Being locally real analytic, $f_{1}$ is real analytic. 
Proposition 2.11. Suppose that E, $F$ are locally convex $\mathbb{K}$-vector spaces, $U$ an open subset of $E$, and $f: U \rightarrow F$ a $\mathbb{K}$-analytic mapping. If $F_{0}$ is a sequentially closed vector subspace of $F$ containing the image of $f$, then $f$ is also $\mathbb{K}$-analytic as a mapping into $F_{0}$.

Proof. As $F_{0}$ is sequentially closed and directional derivatives can be calculated as limits of sequences of difference quotients, we find inductively that $\operatorname{im} d^{n} f \subseteq F_{0}$ for all $n \in \mathbb{N}_{0}$. If $\mathbb{K}=\mathbb{C}$, given $x \in U$ we have $f(x+h)=\sum_{n=0}^{\infty} \frac{1}{n !} \delta_{x}^{n} f(h)$ for $h \in E$ sufficiently small, where $\delta_{x}^{n} f: E \rightarrow F$ is a continuous complex homogeneous polynomial whose image is contained in $F_{0}$ by preceding. Thus $\left.f\right|^{F_{0}}$ is complex analytic. In the real case, given $x \in U$ there exists an open 0-neighbourhood $W$ in $E_{\mathbb{C}}$ such that $x+(W \cap E) \subseteq U$ and $g(y):=\sum_{n=0}^{\infty} \frac{1}{n !}\left(\delta_{x}^{n} f\right)_{\mathbb{C}}(y-x)$ defines a complex analytic function $x+W \rightarrow F_{\mathbb{C}}$ such that $f(y)=g(y)$ for all $y \in x+(W \cap E)$ (cf. [4]). Then im $g \subseteq\left(F_{0}\right)_{\mathbb{C}}$, so that $\left.g\right|^{\left(F_{0}\right)_{\mathbb{C}}}$ is complex analytic by the above. We deduce that $\left.f\right|_{x+(W \cap E)} ^{F_{0}}: x+(W \cap E) \rightarrow F_{0}$ is real analytic. Being a locally real analytic mapping, $\left.f\right|^{F_{0}}$ is real analytic.

REMARK 2.12. It does not seem to be clear whether every complex analytic mapping $f: U \rightarrow F$ as in Definition 2.1 is also real analytic in the sense of Definition 2.3 when $E$ and $F$ are considered as real locally convex spaces (unless $E$ and $F$ are Fréchet spaces). However, the author doesn't know of any counterexample.

REMARK 2.13. Suppose that a continuous mapping $f: U \rightarrow F$ from an open zeroneighbourhood $U$ in a complex locally convex space $E$ to another complex locally convex space $F$ has the form $f(x)=\sum_{n=0}^{\infty} \beta_{n}(x)$ for certain continuous homogeneous polynomials $\beta_{n}$ over $\mathbb{C}$ of degree $n$. Then $f$ is complex analytic as a mapping into the completion $\bar{F}$ of $F$ by [4], Theorem 5.1. The author believes that, unless $F$ is sequentially complete (in which case the cited theorem applies), one should not expect that $f$ is complex analytic as a mapping into $F$. However, explicit counterexamples do not seem to be known.

3. Infinite-dimensional Lie groups and manifolds. Having generalized all basic results of differential calculus from the sequentially complete case to the general case, we can proceed line by line as Milnor [15] to define and study smooth (resp., real analytic, resp., complex analytic) manifolds modelled on arbitrary locally convex spaces (instead of s.c.l.c. spaces); their tangent manifolds; vector fields on manifolds; and smooth (resp., real analytic, resp., complex analytic) Lie groups modelled on arbitrary locally convex spaces.

We shall not give any proofs, since all arguments would be identical with Milnor's.

Definition 3.1. A smooth (resp., real analytic, complex analytic, $\mathcal{C}^{r}$ ) manifold, modelled on a locally convex space $V$, is a Hausdorff topological space $M$, together with a (maximal) atlas of local charts $\kappa: M_{\kappa} \rightarrow V_{\kappa}$ taking an open subset $M_{\kappa} \subseteq M$ homeomorphically to an open subset $V_{\kappa} \subseteq V$, such that all transition maps are smooth mappings (resp., real analytic, complex analytic, of class $\left.\mathcal{C}^{r}\right){ }^{3}$

\footnotetext{
${ }^{3}$ The reader may wish to follow Milnor and require in addition that $M$ be a regular topological space.
} 
Only smooth manifolds, real analytic manifolds, and complex analytic manifolds shall be considered in the following.

3.2. We refer to ([15], notably Section 4) for the definition of the (geometric) tangent space $T_{x} M \cong V$ to the manifold $M$ at $x \in M$, the tangent manifold $T M$, the definition of smooth, real analytic, and complex analytic mappings $\phi: M_{1} \rightarrow M_{2}$ between the corresponding types of manifolds, and the tangent maps $T_{x} \phi: T_{x} M_{1} \rightarrow T_{\phi(x)} M_{2}$. Also Milnor's definition and discussions of vector fields carry over word by word to the setting of manifolds modelled on general locally convex spaces, and we shall not repeat them here.

Definition 3.3. A smooth (resp., real analytic, resp., complex (analytic)) Lie group is a group $G$, equipped with a smooth (resp., real analytic, resp., complex analytic) manifold structure modelled on a locally convex space $V$ such that the inversion map $G \rightarrow G$ and group multiplication $G \times G \rightarrow G$ are smooth (resp., real analytic, resp., complex analytic) mappings (with the apparent manifold structure on the product). The Lie algebra of $G$ is the tangent space $L(G):=T_{1}(G) \cong V$ at the identity element, equipped with the real (resp., complex) topological Lie algebra structure described in [15], Section 5 .

REMARK 3.4. Due to the possible pathologies described in Remark 2.12 above, it might happen that a complex analytic Lie group $G$ cannot be turned into a real analytic Lie group by forgetting the complex structure. In particular, it wouldn't make sense to speak of real analytic mappings or homomorphisms into $G$ in such a case. However, any real or complex analytic Lie group $G$ has an underlying smooth Lie group structure (cf. Proposition 2.4). In particular, we may speak of smooth homomorphism into $G$.

REMARK 3.5. Occasionally, we shall find it convenient to refer to " $\mathbb{K}$-analytic"-Lie groups, manifolds or mappings instead of real, resp., complex analytic ones, where $\mathbb{K} \in$ $\{\mathbb{R}, \mathbb{C}\}$. If we speak of "real Lie groups," it will be clear from the context whether we refer to smooth Lie groups, real analytic Lie groups, or both.

Let us adapt further standard notations and facts from [15] to our more general setting.

Definition 3.6. If $f: G \rightarrow H$ is a smooth homomorphism between smooth Lie groups, we define $L(f):=T_{1}(f): L(G) \rightarrow L(H)$. Then $L(f)$ is a continuous real Lie algebra homomorphism, and a complex Lie algebra homomorphism if $G$ and $H$ are complex Lie groups and $f$ is complex analytic (cf. [15], Section 7). We write $\operatorname{Ad}_{g}:=L\left(I_{g}\right) \in$ $\operatorname{Aut}(L(G))$ for $g \in G$, where $I_{g}: G \rightarrow G, x \mapsto g x g^{-1}$. A one-parameter subgroup of a Lie group $G$ is a smooth homomorphism $\gamma: \mathbb{R} \rightarrow G$. For every $X \in L(G)$, there is at most one one-parameter subgroup $\gamma_{X}$ such that $\gamma_{X}^{\prime}(0)=X$ (cf. [15], Section 7). If $\gamma_{X}$ exists for each $X \in L(G)$, we define the exponential function of $G$ as the mapping

$$
\exp _{G}: L(G) \rightarrow G, \quad X \mapsto \gamma_{X}(1) .
$$

Note that we only speak of an exponential function if the latter is defined on all of $L(G)$.

REMARK 3.7. If $G$ has an exponential function, then $\gamma_{X}=\exp _{G}(\bullet X)$ for all $X \in$ $L(G)$. Since $f \circ \gamma_{X}$ is a one-parameter subgroup of $H$ such that $\left(f \circ \gamma_{X}\right)^{\prime}(0)=T_{1} f \cdot \gamma_{X}^{\prime}(0)=$ 
$L(f) . X$ for each $X \in L(G)$ if $f: G \rightarrow H$ is a smooth homomorphism between Lie groups possessing exponential functions, we have $\exp _{H} \circ L(f)=f \circ \exp _{G}$ in this case ("naturality of exp").

REMARK 3.8. A Lie group modelled on a non-sequentially complete space need not have an exponential function (see [9]). If it exists, the exponential function might fail to be smooth (resp., $\mathbb{K}$-analytic) in pathological cases. It is known that, in general, the exponential function need not induce a local diffeomorphism at 0 : it may fail to be locally injective at 0 , and the exponential image may fail to be an identity neighbourhood. Direct limits of suitable ascending sequences of finite-dimensional Lie groups provide simple examples for such behaviour ([7], Example 5.5).

REMARK 3.9. Although, as we have seen, sequential completeness of the modelling spaces doesn't play a role for most of the basic constructions of Lie theory, of course there are specific questions where sequential completeness cannot be dispensed with. For example, the central extension theory for infinite-dimensional Lie groups (see, e.g., [17]) depends vitally on Poincaré's Lemma, whose proof makes use of sequential completeness.

4. BCH-Lie groups, their Lie quotients and universal complexifications. In this section, we define a class of well-behaved Lie groups, the Baker-Campbell-Hausdorff (BCH-) Lie groups, which share important properties of Banach-Lie groups. A convincing solution to the existence problem of Lie group structures on quotients of BCH-Lie groups, as well as a convincing solution to the existence problem of universal complexifications of BCH-Lie groups, depend essentially on the availability of Lie groups modelled on arbitrary locally convex spaces, not only on sequentially complete ones. We present the solutions to these problems, and explain why Lie groups modelled on sequentially complete spaces would not be sufficient.

Definition 4.1. A real or complex analytic Lie group $G$ modelled on a locally convex space is called a Baker-Campbell-Hausdorff (BCH-) Lie group if it has the following properties:

(a) $G$ has an exponential function $\exp _{G}: L(G) \rightarrow G$ (defined on all of $L(G)$ ), and there is an open zero-neighbourhood $U$ in $L(G)$ such that $V:=\exp _{G}(U)$ is open in $G$ and $\phi:=\left.\exp _{G}\right|_{U} ^{V}: U \rightarrow V$ is a diffeomorphism of real (resp., complex) analytic manifolds.

(b) There is an open zero-neighbourhood $W \subseteq U$ in $L(G)$ such that $\exp _{G}(W) \exp _{G}(W)$ $\subseteq \exp _{G}(U)$ and $\phi^{-1}(\phi(X) \phi(Y))=\sum_{n=1}^{\infty} \beta_{n}(X, Y)=: X * Y$ is given by the Campbell-Hausdorff series for $X, Y \in W$ (with pointwise convergence).

Thus $\beta_{1}(X, Y)=X+Y, \beta_{2}(X, Y)=\frac{1}{2}[X, Y], \beta_{3}(X, Y)=\frac{1}{12}([X,[X, Y]]+[Y,[Y, X]])$, etc. Note that every $\beta_{n}$ is a continuous homogeneous polynomial, the Lie bracket being continuous (cf. [15], p. 1037).

ExAMPLE 4.2. Every Banach-Lie group is a BCH-Lie group.

EXAmPle 4.3. Let $M$ be a $\sigma$-compact, finite-dimensional smooth manifold, $0 \leq r \leq$ $\infty$, and $G$ be a $\mathbb{K}$-analytic BCH-Lie group. Then the mapping group $\mathcal{C}_{K}^{r}(M, G):=\{\gamma \in$ 
$\left.\mathcal{C}^{r}(M, G):\left.\gamma\right|_{M \backslash K}=1\right\}$ can be made a $\mathbb{K}$-analytic BCH-Lie group with Lie algebra $\mathcal{C}_{K}^{r}(M, L(G))$, for every compact subset $K$ of $M$ ([8]; cf. [15] when $M=K$ and $G$ is finite-dimensional). Also the test function groups $\mathcal{D}^{r}(M, G)=\bigcup_{K} \mathcal{C}_{K}^{r}(M, G)$ (as defined in [1] when $G$ is finite-dimensional) admit natural $\mathbb{K}$-analytic BCH-Lie group structures, modelled on the locally convex direct limit $\mathcal{D}^{r}(M, L(G))=\lim _{\longrightarrow} \mathcal{C}_{K}^{r}(M, L(G)$ ) (see [8], Section 4).

ExAmPle 4.4. Prominent examples of direct limits of finite-dimensional Lie groups, as $\mathrm{GL}_{\infty}(\mathbb{R})$ or $\mathrm{SL}_{\infty}(\mathbb{R})$ (see [16], [7]) are real BCH-Lie groups modelled on non-metrizable spaces (see [8]). More generally, all analytic subgroups of $\mathrm{GL}_{\infty}(\mathbb{R})$ (called "linear direct limit Lie groups" in [8]) are real BCH-Lie groups ([8]; cf. [14], Theorem 47.9). However, pathological examples of direct limit Lie groups (as [7], Example 5.5) fail to be BCH.

EXAMPLE 4.5. The diffeomorphism group $\operatorname{Diff}(M)$ of any connected, non-singleton, compact smooth manifold $M$ is not a $\mathrm{BCH}$-Lie group, as it cannot be given a real analytic structure ([15], Corollary 9.2).

REMARK 4.6. The theory of BCH-Lie groups is elaborated in [8], on the foundation of the differential calculus developed in the present article. In particular, as in the Banach case it can be shown that every continuous homomorphism between real BCH-Lie groups is real analytic (and every continuous homomorphism between complex BCH-Lie groups is smooth). As a consequence, real BCH-Lie groups can be identified with the underlying smooth Lie groups (or even the underlying topological groups).

We recall the definition of Lie subgroups:

Definition 4.7. A $\mathbb{K}$-analytic BCH-Lie group $H$ is called a $\mathbb{K}$-Lie subgroup of a $\mathbb{K}$-analytic BCH-Lie group $G$ if the topological group underlying $H$ is a subgroup of $G$, equipped with the induced topology, and $L(\varepsilon)$ is $\mathbb{K}$-linear, where $\varepsilon: H \hookrightarrow G$ is the inclusion map.

REMARK 4.8. Note that $\varepsilon$ is $\mathbb{K}$-analytic in the preceding situation, and $L(\varepsilon)$ is a topological embedding. We use the latter embedding to identify $L(H)$ with its image in $L(G)$, which is $\left\{X \in L(G): \exp _{G}(\mathbb{R} X) \subseteq H\right\}=: \mathfrak{h}$. Using this identification, the exponential function of $H$ is $\left.\exp _{G}\right|_{\mathfrak{h}}$.

Definition 4.9. If $G$ is a $\mathbb{K}$-analytic BCH-Lie group and $H$ a sequentially closed subgroup of $G$, we set $L(H):=\left\{X \in L(G): \exp _{G}(\mathbb{R} X) \subseteq H\right\}$.

REMARK 4.10. It can be shown that $L(H)$ is a real Lie subalgebra of $L(G)$, and a real Lie algebra ideal if $H$ is a sequentially closed normal subgroup of $G$ ([8], Section 2).

Universal complexifications are defined as follows:

Definition 4.11. Let $\mathcal{A}$ be a full subcategory of the category of complex Lie groups and complex analytic homomorphisms. Given a smooth real Lie group $G$, we say that a complex Lie group $G_{\mathbb{C}} \in$ ob $\mathcal{A}$, together with a smooth homomorphism $\gamma_{G}: G \rightarrow G_{\mathbb{C}}$, is a universal complexification of $G$ in $\mathcal{A}$ if for every smooth homomorphism $\phi: G \rightarrow$ $H$ from $G$ to a complex Lie group $H \in$ ob $\mathcal{A}$, there exists a unique complex analytic homomorphism $\tilde{\phi}: G_{\mathbb{C}} \rightarrow H$ such that $\tilde{\phi} \circ \gamma_{G}=\phi$. 
We recall the concept of enlargibility:

Definition 4.12. A locally convex topological $\mathbb{K}$-Lie algebra is called enlargible if it is isomorphic to the Lie algebra of some $\mathbb{K}$-analytic BCH-Lie group.

The following two theorems initiated the studies presented here. The first of these is [8], Theorem 2.20, which generalizes the Banach case treated before in [10], Theorem II.2.

ThEOREM 4.13 (Quotient Theorem). Let $G$ be a $\mathbb{K}$-analytic BCH-Lie group with Lie algebra $\mathfrak{g}, N$ be a closed normal subgroup of $G$, and $\mathfrak{n}:=\left\{X \in \mathfrak{g}: \exp _{G}(\mathbb{R} X) \subseteq N\right\}$. If $\mathbb{K}=\mathbb{C}$, we assume furthermore that $\mathfrak{n}$ is a complex subalgebra of $L(G)$. Let $q: G \rightarrow G / N$, $Q: \mathfrak{g} \rightarrow \mathfrak{g} / \mathfrak{n}$ be the canonical quotient maps. Then the following conditions are equivalent:

(a) There exists a smooth (resp., complex analytic) homomorphism $\phi: G \rightarrow H$ into a $\mathbb{K}$-Lie group ${ }^{4} H$ whose exponential function is locally injective at zero, such that $\operatorname{ker}(\phi)=N$.

(b) $G / N$ can be made a $\mathbb{K}$-analytic BCH-Lie group with Lie algebra $\mathfrak{g} / \mathfrak{n}$, such that $q \circ \exp _{G}=\exp _{G / N} \circ Q$.

(c) $N$ is a $\mathbb{K}$-Lie subgroup of $G$.

The second result essentially depending on the availability of Lie groups modelled on non-sequentially complete spaces is the following characterization of BCH-Lie groups admitting universal complexifications:

THEOREM 4.14 (Complexification Theorem). Given a real BCH-Lie group G, let $N$ be the intersection of all kernels of smooth homomorphisms from G into complex BCH-Lie groups. Then the following conditions are equivalent:

(a) $G$ has a universal complexification $G_{\mathbb{C}}$ in the category of complex BCH-Lie groups.

(b) $N$ is a Lie subgroup of $G$, and $L(G / N)_{\mathbb{C}} \cong(L(G) / L(N))_{\mathbb{C}}$ is enlargible.

If these conditions are satisfied, then $L\left(G_{\mathbb{C}}\right)=(L(G) / L(N))_{\mathbb{C}}$. In particular, $G_{\mathbb{C}}$ is a Banach-Lie group (resp., BCH-Fréchet-Lie group) if $G$ is so.

Proof. This is a special case of [8], Theorem 7.2.

REMARK 4.15. An analogous characterization of Banach-Lie groups with universal complexifications in the category of complex Banach-Lie groups was obtained earlier in $[10]$.

REMARK 4.16. Suppose that, on the basis of Milnor's concept of Lie groups modelled on s.c.l.c. spaces, we had made sequential completeness of the modelling locally convex spaces part of the definition of BCH-Lie groups. In this case, whenever $L(G) / L(N)$ fails to be sequentially complete for a closed normal Lie subgroup $N$ of $G$, the topological group $G / N$ could not be made a BCH-Lie group in the situation of Theorem 4.13 (although the Campbell-Hausdorff series still describes the group multiplication locally). Furthermore, whenever the corresponding topological Lie algebra $L(G) / L(N)$ in the situation of Theorem 4.14 fails to be sequentially complete, nothing much can be said about the existence of universal complexifications. Both theorems would have to be reformulated

\footnotetext{
${ }^{4}$ Smooth or real analytic in the real case.
} 
along the lines: "If $L(G) / L(N)$ is sequentially complete, then the following conditions are equivalent". The author considers these restrictions as unnatural, depriving the theorems of their natural simplicity, and it was the desire to remove these unnecessary restrictions which inspired the studies performed in the present paper.

We conclude the article with another selected result from [8]:

THEOREM 4.17. If $N=\{1\}$ and $L(G)_{\mathbb{C}}$ is enlargible in the situation of Theorem 4.14, then $G_{\mathbb{C}}$ is the universal complexification of $G$ in the category of all complex Lie groups with complex analytic exponential functions.

Proof. This is a special case of [8], Corollary 7.6.

\section{References}

[1] S. Albeverio, R. J. Høegh-Krohn, J. A. Marion, D. H. Testard, and B. S. Torrésani, Noncommutative Distributions, Marcel Dekker, 1993.

[2] A. Bastiani, Applications différentiables et variétés différentiables de dimension infinie, J. Analyse Math. 13 (1964), 1-114.

[3] J. Bochnak and J. Siciak, Polynomials and multilinear mappings in topological vector spaces, Studia Math. 39 (1971), 59-76.

[4] J. Bochnak and J. Siciak, Analytic functions in topological vector spaces, ibid., 77-112.

[5] H. Bosek, G. Czichowski and K.-P. Rudolph, Analysis on Topological Groups - General Lie Theory, Teubner, Leipzig, 1981.

[6] H. Glöckner, Infinite-Dimensional Complex Groups and Semigroups: Representations of Cones, Tubes, and Conelike Semigroups, Doctoral Dissertation, Darmstadt University of Technology, Department of Mathematics, 2000. Advisor: K.-H. Neeb.

[7] H. Glöckner, Direct limit Lie groups and manifolds, Kyoto J. Math., to appear.

[8] H. Glöckner, Lie group structures on quotient groups and universal complexifications for infinite-dimensional Lie groups, J. Funct. Analysis, to appear.

[9] H. Glöckner, Algebras whose groups of units are Lie groups, submitted.

[10] H. Glöckner and K.-H. Neeb, Banach-Lie quotients, enlargibility, and universal complexifications, LSU preprint 2001-4, April 2001.

[11] R. Hamilton, The inverse function theorem of Nash and Moser, Bull. Amer. Math. Soc. 7 (1982), 65-222.

[12] M. Hervé, Analyticity in Infinite-Dimensional Spaces, de Gruyter, Berlin, 1989.

[13] H. H. Keller, Differential Calculus in Locally Convex Spaces, Springer, 1974.

[14] A. Kriegl and P. W. Michor, The Convenient Setting of Global Analysis, Amer. Math. Soc., Providence R.I., 1997.

[15] J. Milnor, Remarks on infinite dimensional Lie groups, in: B. DeWitt, and R. Stora (Eds.), Relativity, Groups and Topology II, North-Holland, 1983, 1008-1057.

[16] L. Natarajan, E. Rodríguez-Carrington, and J. A. Wolf, Differentiable structure for direct limit groups, Letters in Math. Physics 23 (1991), 99-109.

[17] K.-H. Neeb, Central extensions of infinite-dimensional Lie groups, preprint 2084, TU Darmstadt, April 2000.

[18] E. G. F. Thomas, Calculus on locally convex spaces, preprint W-9604, Univ. of Groningen, 1996. 